ANNALES

POLONICI MATHEMATICI

LXXVIII.3 (2002)

\title{
Functions uniformly quiet at zero and existence results for one-parameter boundary value problems
}

\author{
by G. L. Karakostas and P. Ch. Tsamatos (Ioannina)
}

\begin{abstract}
We introduce the notion of uniform quietness at zero for a real-valued function and we study one-parameter nonlocal boundary value problems for second order differential equations involving such functions. By using the Krasnosel'skii fixed point theorem in a cone, we give values of the parameter for which the problems have at least two positive solutions.
\end{abstract}

1. Introduction. Recently the authors [17] introduced the notion of a real-valued function "quiet at zero", as follows:

A continuous function $f:[0, \infty)=: \mathbb{R}^{+} \rightarrow \mathbb{R}$, with $f(x)>0$ when $x>0$, is said to be quiet at zero if for each $T>0$ there is a $\mu>0$ such that for all $\tau \in[0, T]$,

$$
\sup \{f(x): x \in[0, \tau]\} \leq \mu \inf \{f(x): x \in[\tau, T]\} .
$$

For instance, if $f$ is increasing in a right neighborhood of 0 , or $f(0)>0$, then it is quiet at zero. Also it is easy to see that in (1.1) the constant $\mu$ is always greater than or equal to 1 .

In this paper we extend the above notion by defining functions "uniformly quiet at zero". This is the case when the bound $\mu$ does not depend on $T$ :

Definition. A continuous function $f: \mathbb{R}^{+} \rightarrow \mathbb{R}$, with $f(x)>0$ when $x>0$, will be said to be uniformly quiet at zero if there is a $\mu \geq 1$ such that inequality (1.1) is satisfied for all $T>0$ and all $\tau \in(0, T)$.

It is clear that if $f$ is monotone and $\lim _{x \rightarrow \infty} f(x)>0$, then it is uniformly quiet at zero.

Now consider the following boundary value problem:

$$
\left(\left.1.2\right|_{\lambda}\right) \quad\left(p(t) x^{\prime}\right)^{\prime}+\lambda q(t) f(x)=0, \quad \text { a.e. } t \in[0,1],
$$

2000 Mathematics Subject Classification: Primary 34B18.

Key words and phrases: function quiet and uniformly quiet at zero, nonlocal boundary value problems, two positive solutions, Krasnosel'skiı̌'s fixed point theorem. 


$$
\begin{gathered}
x(0)=0, \\
x^{\prime}(1)=\int_{\eta}^{1} x^{\prime}(s) d g(s),
\end{gathered}
$$

with $f: \mathbb{R}^{+} \rightarrow \mathbb{R}$ being uniformly quiet at zero. The real-valued functions $p, q, g$ are defined at least on the interval $[0,1], \eta \in(0,1)$ and $\lambda$ is a positive parameter. The integral in (1.4) is meant in the sense of Riemann-Stieltjes. Also we assume that $p(1)=1$, as well as $\int_{\eta}^{1}(1 / p(s)) d g(s)<1$ and so

$$
\alpha:=\left(1-\int_{\eta}^{1} \frac{1}{p(s)} d g(s)\right)^{-1}>0 .
$$

This problem is a nonlocal boundary value problem which includes as special cases multipoint boundary value problems considered by several authors during the last decade. More details about this problem can be found e.g. in the papers [16-19] and in their references.

Our purpose here is to show that under the assumption that $f$ is uniformly quiet at zero and satisfies some rather mild conditions, there are at least two positive solutions for the above boundary value problem. The most appropriate tool, which is used to guarantee both the existence of solutions as well as their positivity, is the following well known theorem due to Krasnosel'skii [20].

TheOREM 1.1. Let $\mathcal{B}$ be a Banach space and let $\mathbb{K}$ be a cone in $\mathcal{B}$. Assume that $\Omega_{1}, \Omega_{2}$ are open subsets of $\mathcal{B}$, with $0 \in \Omega_{1} \subset \operatorname{cl} \Omega_{1} \subset \Omega_{2}$, and let

$$
A: \mathbb{K} \cap\left(\operatorname{cl} \Omega_{2} \backslash \Omega_{1}\right) \rightarrow \mathbb{K}
$$

be a completely continuous operator such that either

$$
\|A u\| \leq\|u\|, \quad u \in \mathbb{K} \cap \partial \Omega_{1}, \quad\|A u\| \geq\|u\|, \quad u \in \mathbb{K} \cap \partial \Omega_{2},
$$

or

$$
\|A u\| \geq\|u\|, \quad u \in \mathbb{K} \cap \partial \Omega_{1}, \quad\|A u\| \leq\|u\|, \quad u \in \mathbb{K} \cap \partial \Omega_{2} .
$$

Then $A$ has a fixed point in $\mathbb{K} \cap\left(\operatorname{cl} \Omega_{2} \backslash \Omega_{1}\right)$.

In this theorem $\partial \Omega$ denotes the boundary and $\operatorname{cl} \Omega$ the closure of the set $\Omega$. We follow the technique in $[16,17]$ and apply this theorem twice, so that our conclusion follows. The results here are motivated mainly by the papers [15-17].

The paper is organized as follows. In Section 2 we elaborate a little on the notion of a function uniformly quiet at zero. Some examples are also given to illustrate this concept. In Section 3, by using the notion of uniform quietness at zero, we find neighborhoods of $\lambda$ in which the boundary value 
problem $\left(\left.1.2\right|_{\lambda}\right),(1.3),(1.4)$ has at least two positive solutions, under various conditions.

2. Some facts about quietness at zero. If $f$ is uniformly quiet at zero, then it is quiet at zero, but not conversely. We present two examples.

(a) Let $f$ a real-valued continuous function on $[0, \infty)$, with $f(x)>0$, defined as follows: Its graph passes through the points $(1 /(2 n), 1 / \sqrt{2 n})$ and $(1 /(2 n+1), 1 /(2 n+1))$ of the plane, for all $n \geq 1$, and it is linear between these points. If for $T=1$ there is a $\mu \geq 1$ such that condition (1.1) holds, then, for all large $n$, we must have

$$
\frac{1}{\sqrt{2 n}} \leq \mu \frac{1}{2 n-1},
$$

which is a contradiction. This means that $f$ is not quiet at zero.

(b) Consider a real function $f$ defined at least on $[0, \infty)$, with $f(x)>0$ when $x>0, f(0)=0, f(2 k+1)=2 k+1$ for $k=0,1,2, \ldots, f(2 k)=4 k^{2}$ for $k=1,2, \ldots$ and linear in-between. It is increasing on $[0,2]$, thus it is quiet at zero. If $f$ were uniformly quiet at zero, then there would exist a $\mu \geq 1$ satisfying (1.1). Hence, for each $k$ large enough and $T>2 k$,

$$
\sup \{f(x): x \in[0,2 k]\} \leq \mu \inf \{f(x): x \in[2 k, T]\},
$$

and so $4 k^{2} \leq \mu(2 k+1)$, a contradiction. Thus $f$ is not uniformly quiet at zero.

A function uniformly quiet at zero has the following characteristic:

Proposition 2.1. Let $f$ be a function uniformly quiet at zero and let $q:[0,1] \rightarrow \mathbb{R}^{+}$be a continuous function not identically zero. Then, for each $r \in(0,1)$ and all increasing functions $u:[0,1] \rightarrow \mathbb{R}^{+}$,

$$
\int_{0}^{1} q(s) f(u(s)) d s \leq \xi_{r} \int_{r}^{1} q(s) f(u(s)) d s,
$$

where $\xi_{r}$ is the constant defined by

$$
\xi_{r}:=\frac{\int_{0}^{r} q(s) d s}{\int_{r}^{1} q(s) d s} \mu+1 .
$$

Proof. Let $\mu \geq 1$ be a constant satisfying (1.1) for all $T>0$ and $\tau \in$ $(0, T)$. Let also $r \in(0,1)$ be fixed and consider any increasing function $u:[0,1] \rightarrow \mathbb{R}^{+}$. We set $u(1)=: T$ and $u(r)=: \tau$. Since $u$ is increasing, we have $\tau \leq T$. Moreover we observe that 


$$
\begin{aligned}
\int_{0}^{1} q(s) f(u(s)) d s=\int_{0}^{r} q(s) f(u(s)) d s+\int_{r}^{1} q(s) f(u(s)) d s \\
\leq \sup _{w \in[0, \tau]} f(w) \int_{0}^{r} q(s) d s+\int_{r}^{1} q(s) f(u(s)) d s \\
\leq \quad \frac{\int_{0}^{r} q(s) d s}{\int_{r}^{1} q(s) d s} \cdot \frac{\sup _{w \in[0, \tau]} f(w)}{\inf _{w \in[\tau, T]} f(w)} \int_{r}^{1} q(s) f(u(s)) d s \\
\quad+\int_{r}^{1} q(s) f(u(s)) d s \\
\leq\left(\frac{\int_{0}^{r} q(s) d s}{\int_{r}^{1} q(s) d s} \mu+1\right) \int_{r}^{1} q(s) f(u(s)) d s=\xi_{r} \int_{r}^{1} q(s) f(u(s)) d s
\end{aligned}
$$

If $f$ is increasing, then inequality (2.1) proved in Proposition 2.1 holds with $\mu=1$ and $\xi_{r}=\int_{0}^{1} q(s) d s / \int_{r}^{1} q(s) d s$. And this constant $\xi_{r}$ is the best possible: set $u(t)=$ constant.

\section{Existence of two solutions of the boundary value problem} $\left(\left.1.2\right|_{\lambda}\right),(1.3),(1.4)$. In this section we investigate the existence of positive solutions of the boundary value problem $\left(\left.1.2\right|_{\lambda}\right),(1.3),(1.4)$. In most studies of the existence of positive solutions for such second order boundary value problems, a common assumption is the existence of the limit of the fraction $f(u) / u$ as $u$ approaches 0 and $\infty$ (see [1-15]). Here, we weaken these assumptions and use the lower and upper limits at these points. Our purpose is to define an interval of the real line for the parameter $\lambda$ so that the corresponding boundary value problem $\left(\left.1.2\right|_{\lambda}\right),(1.3),(1.4)$ has at least two positive solutions.

Denote by $I$ the interval $[0,1]$, and by $C(I)$ the space of all continuous functions $x: I \rightarrow \mathbb{R}$. Let $C_{0}^{1}(I)$ be the space of all functions $x: I \rightarrow \mathbb{R}$ whose first derivative $x^{\prime}$ is absolutely continuous on $I$ and $x(0)=0$. This is a Banach space with the norm \| $\|$ defined by

$$
\|x\|:=\sup \left\{\left|x^{\prime}(t)\right|: t \in I\right\} .
$$

Also, we denote by $L_{1}^{+}(I)$ the space of all functions $x: I \rightarrow \mathbb{R}^{+}$which are Lebesgue integrable on $I$, endowed with its usual norm \|\|$_{1}$.

By a solution of the problem $\left(\left.1.2\right|_{\lambda}\right),(1.3),(1.4)$ we mean a function $x \in C_{0}^{1}(I)$ satisfying $(1.4)$ and $\left(\left.1.2\right|_{\lambda}\right)$ for almost all $t \in I$.

As indicated in $[16,17]$, the problem $\left(\left.1.2\right|_{\lambda}\right),(1.3),(1.4)$ is equivalent to the operator equation $x=A_{\lambda} x, x \in C_{0}^{1}(I)$, where $A_{\lambda}$ is the operator 
defined by

$$
A_{\lambda} x(t):=\lambda \alpha P(t) \int_{\eta}^{1} \Phi(f(x))(s) d g(s)+\lambda \int_{0}^{t} \Phi(f(x))(s) d s,
$$

where we have set

$$
\begin{aligned}
P(t) & :=\int_{0}^{t} \frac{1}{p(s)} d s, \quad t \in I, \\
\Phi(y)(t) & :=\frac{1}{p(t)} \int_{t}^{1} q(s) y(s) d s, \quad t \in I, y \in C(I) .
\end{aligned}
$$

Notice that $\alpha$ is the number defined in (1.5). We also set

$$
D:=\int_{\eta}^{1} \Phi(P)(s) d g(s) \text { and } H:=\int_{\eta}^{1} \Phi(1)(s) d g(s) .
$$

It is clear that $A_{\lambda}$ is a completely continuous operator. Now we present the list of assumptions which we use in the following.

(H1) $\quad f: \mathbb{R} \rightarrow \mathbb{R}$ is a continuous function, with $f(x) \geq 0$ when $x>0$, and uniformly quiet at zero.

(H2) The functions $p, q$ are continuous on $I$ and such that $p>0, q \geq 0$ and $\sup \{q(s): \eta \leq s \leq 1\}>0$. We assume that $p$ is nondecreasing.

(H3) The function $g: I \rightarrow \mathbb{R}$ is increasing and $g(\eta)=0<g(\eta+)=: b_{0}$.

If $\mu \geq 1$ and $\xi_{\eta}$ are the constants introduced by Proposition 2.1 (due to assumption (H1)), we set

$$
\begin{gathered}
\Lambda:=\xi_{\eta} \sup _{s \in I} p(s), \quad b:=\min \left\{b_{0}, \frac{\Lambda H}{\alpha|D \eta p(0)-H|}\right\}, \\
\sigma:=\frac{\alpha b D p(0)}{\alpha b+\Lambda}, \quad \theta:=\frac{p(0)}{\alpha H+\|q\|_{1}} .
\end{gathered}
$$

We observe that $\sigma \eta \leq H$ (see also [17]) and therefore the function

$$
f_{i}(w):=\inf \left\{f(z): \frac{\eta \sigma}{H} w \leq z \leq w\right\}, \quad w>0,
$$

is well defined. We also consider the function

$$
f_{s}(w):=\sup \{f(x): x \in[0, w]\}, \quad w>0 .
$$

Next define the set

$\mathbb{K}:=\left\{x \in C_{0}^{1}(I): x \geq 0, x^{\prime} \geq 0, x\right.$ is concave and $\left.\int_{\eta}^{1} \Phi(x)(s) d g(s) \geq \sigma\|x\|\right\}$, which, obviously, is a cone in $C_{0}^{1}(I)$. 
LEMMA 3.1. If $x$ is a continuous, increasing and nonnegative function on $I$, then

$$
\int_{\eta}^{1} \Phi(f(x))(s) d g(s) \geq \frac{b_{0}}{\Lambda} \int_{0}^{1} q(s) f(x(s)) d s .
$$

Proof. From assumption (H3) we have

$$
g(s) \geq b_{0}, s \in(\eta, 1] .
$$

By using this inequality and Proposition 2.1 we get

$$
\begin{aligned}
\int_{0}^{1} q(s) f(x(s)) d s \leq \xi_{\eta} \int_{\eta}^{1} q(s) f(x(s)) d s \leq \frac{\xi_{\eta}}{b_{0}} \int_{\eta}^{1} q(s) f(x(s)) g(s) d s \\
=-\frac{\xi_{\eta}}{b_{0}} \int_{\eta}^{1} d\left(\int_{s}^{1} q(r) f(x(r)) d r\right) g(s)=\frac{\xi_{\eta}}{b_{0}} \int_{\eta}^{1} \int_{s}^{1} q(r) f(x(r)) d r d g(s) \\
\leq \frac{\Lambda}{b_{0}} \int_{\eta}^{1} \frac{1}{p(s)} \int_{s}^{1} q(r) f(x(r)) d r d g(s)=\frac{\Lambda}{b_{0}} \int_{\eta}^{1} \Phi(f(x))(s) d g(s) .
\end{aligned}
$$

Lemma 3.2. Assume that assumptions (H1), (H2) hold and let $\lambda>0$. Then

$$
A_{\lambda} \mathbb{K} \subset \mathbb{K}
$$

Proof. We observe that $A_{\lambda} x \geq 0,\left(A_{\lambda} x\right)^{\prime} \geq 0$ and $A_{\lambda} x$ is concave for all $x \in \mathbb{K}$. Moreover, from (3.1) we have

$$
\begin{aligned}
\int_{\eta}^{1} \Phi\left(A_{\lambda} x\right)(s) d g(s) \geq & \lambda \alpha \int_{\eta}^{1} \Phi(P)(s) d g(s) \int_{\eta}^{1} \Phi(f(x))(s) d g(s) \\
= & \lambda \alpha D \int_{\eta}^{1} \frac{1}{p(s)} \int_{s}^{1} q(r) f(x(r)) d r d g(s) \\
= & \lambda \frac{\sigma\left(\alpha b_{0}+\Lambda\right)}{b_{0} p(0)} \int_{\eta}^{1} \frac{1}{p(s)} \int_{s}^{1} q(r) f(x(r)) d r d g(s) \\
= & \lambda \frac{\sigma}{p(0)}\left(\alpha+\frac{\Lambda}{b_{0}}\right)^{1} \int_{\eta}^{1} \frac{1}{p(s)} \int_{s}^{1} q(r) f(x(r)) d r d g(s) \\
= & \lambda \sigma \frac{\alpha}{p(0)} \int_{\eta}^{1} \frac{1}{p(s)} \int_{s}^{1} q(r) f(x(r)) d r d g(s) \\
& +\lambda \sigma \frac{1}{p(0)} \cdot \frac{\Lambda}{b_{0}} \int_{\eta}^{1} \frac{1}{p(s)} \int_{s}^{1} q(r) f(x(r)) d r d g(s) .
\end{aligned}
$$


Hence, taking into account Lemma 3.1 we get

$$
\begin{aligned}
\int_{\eta}^{1} \Phi\left(A_{\lambda} x\right)(s) d g(s) \geq & \lambda \sigma \frac{\alpha}{p(0)} \int_{\eta}^{1} \frac{1}{p(s)} \int_{s}^{1} q(r) f(x(r)) d r d g(s) \\
& +\lambda \sigma \frac{1}{p(0)} \int_{0}^{1} q(r) f(x(r)) d r \\
= & \sigma\left(A_{\lambda} x\right)^{\prime}(0)=\sigma\left\|\left(A_{\lambda} x\right)\right\| .
\end{aligned}
$$

Now consider the constants

$$
\begin{gathered}
\beta:=\sup _{u>0} \frac{f_{i}(u)}{u}, \quad l_{0}:=\liminf _{u \rightarrow 0+} \frac{f_{s}(u)}{u}, \quad l_{\infty}:=\liminf _{u \rightarrow \infty} \frac{f_{s}(u)}{u}, \\
\varrho:=\frac{1}{\alpha H}, \quad l:=\theta \min \left\{l_{0}^{-1}, l_{\infty}^{-1}\right\}
\end{gathered}
$$

and we are ready to prove our first main result.

THEOREM 3.3. Suppose that the functions $f, p, q$ and $g$ satisfy assumptions $(\mathrm{H} 1)-(\mathrm{H} 3)$. If $\varrho<l \beta$, then for all $\lambda$ in the interval $(\varrho / \beta, l)$ the boundary value problem $\left(\left.1.2\right|_{\lambda}\right),(1.3),(1.4)$ has at least two positive solutions.

Proof. We set $F:=\lambda f, F_{i}:=\lambda f_{i}$ and $F_{s}:=\lambda f_{s}$. From $\lambda>\varrho / \beta$ we have $\beta>\varrho / \lambda$ and so there exists $u>0$ such that $f_{i}(u) / u>\varrho / \lambda$. This $u$ satisfies $F_{i}(u)>\varrho u$.

Consider a function $x \in \mathbb{K}$ with $\|x\|=u$. The fact that $x$ is concave implies that

$$
\eta x(1) \leq x(\eta) \leq x(r) \leq x(1) \leq\|x\| \quad \text { for every } r \in[\eta, 1] .
$$

So,

$$
\begin{aligned}
\sigma\|x\| & \leq \int_{\eta}^{1} \Phi(x)(s) d g(s)=\int_{\eta}^{1} \frac{1}{p(s)} \int_{s}^{1} q(r) x(r) d r d g(s) \\
& \leq x(1) \int_{\eta}^{1} \frac{1}{p(s)} \int_{s}^{1} q(r) d r d g(s)=x(1) \int_{\eta}^{1} \Phi(1)(s) d g(s)=x(1) H .
\end{aligned}
$$

Thus we have $x(1) \geq \sigma\|x\| / H$, which implies that

$$
x(r) \geq \eta \sigma\|x\| / H, \quad r \in[\eta, 1] .
$$

Then, for every $r \in[\eta, 1]$ we get $\eta \sigma\|x\| / H \leq x(r) \leq\|x\|$. Therefore, since $F_{i}(u)>\varrho u$, we obtain

$$
\left(A_{\lambda} x\right)^{\prime}(1) \geq \alpha \int_{\eta}^{1} \frac{1}{p(s)} \int_{s}^{1} q(r) F(x(r)) d r d g(s) \geq \alpha F_{i}(u) H>\alpha H \varrho u=u .
$$

This means that if $\|x\|=u$, then $\left\|A_{\lambda} x\right\|>\|x\|$. 
On the other hand, from $\lambda<l \leq \theta / l_{\infty}$, we have $\lambda l_{\infty}<\theta$. Hence, there exists $v>u$ such that $F_{s}(v)<\theta v$.

Consider a function $x \in \mathbb{K}$ with $\|x\|=v$. Since $0 \leq x(t) \leq v$ for $t \in I$ and $F_{s}(v)<\theta v$ we have

$$
\begin{aligned}
\left\|A_{\lambda} x\right\|=\left(A_{\lambda} x\right)^{\prime}(0) & =\frac{\alpha}{p(0)} \int_{\eta}^{1} \Phi(F(x))(s) d g(s)+\frac{1}{p(0)} \int_{0}^{1} q(s) F(x(s)) d s \\
& \leq F_{s}(v)\left[\frac{\alpha H}{p(0)}+\frac{1}{p(0)} \int_{0}^{1} q(s) d s\right] \\
& <\theta v\left[\frac{\alpha H+\|q\|_{1}}{p(0)}\right]=v=\|x\| .
\end{aligned}
$$

Finally we set $\Omega_{1}:=\left\{x \in C_{0}^{1}(I):\|x\|<u\right\}$ and $\Omega_{2}:=\left\{x \in C_{0}^{1}(I)\right.$ : $\|x\|<v\}$ and apply Theorem 1.1 by taking into account Lemma 3.2, the fact that $A_{\lambda}$ is a completely continuous operator and $0<u<v$. Then we conclude that there exists a solution $x_{1} \in \mathbb{K}$ of the boundary value problem $\left(\left.1.2\right|_{\lambda}\right),(1.3),(1.4)$ such that $u<\left\|x_{1}\right\|<v$.

Now, since $\lambda<l \leq \theta / l_{0}$ or, equivalently, $\lambda l_{0}<\theta$, there exists $w \in(0, u)$ such that

$$
0 \leq F_{s}(w)<\theta w .
$$

Fix a function $x \in \mathbb{K}$ with $\|x\|=w$. Then $0 \leq x(r) \leq w$ for $r \in I$ and (3.3) works for $w$ in place of $v$, because of (3.4). Thus we get $\left\|A_{\lambda} x\right\| \leq\|x\|$ whenever $\|x\|=w$.

We take into account Lemma 3.2 and the fact that $0<w<u$, and we apply Theorem 1.1 to conclude that there is a solution $x_{2} \in \mathbb{K}$ of the boundary value problem $\left(\left.1.2\right|_{\lambda}\right),(1.3),(1.4)$ such that $w<\left\|x_{2}\right\|<u$.

Corollary 3.4. Consider the functions $f, p, q$ and $g$ satisfying assumptions $(\mathrm{H} 1)-(\mathrm{H} 3)$. Moreover assume that

$$
\varrho<\beta, l_{0}<\theta \text {, and } l_{\infty}<\theta .
$$

Then the boundary value problem $\left(\left.1.2\right|_{1}\right),(1.3),(1.4)$ has at least two positive solutions.

Proof. From (H4) we can easily see that $\varrho / \beta<1<l$ and therefore Theorem 3.3 applies with $\lambda=1$.

Next consider the quantities

$$
B:=\inf _{u>0} \frac{f_{s}(u)}{u}, \quad L_{0}:=\limsup _{u \rightarrow 0+} \frac{f_{i}(u)}{u}, \quad L_{\infty}:=\limsup _{u \rightarrow+\infty} \frac{f_{i}(u)}{u}
$$

and set

$$
\zeta:=\varrho \max \left\{L_{0}^{-1}, L_{\infty}^{-1}\right\}
$$


We have the following theorem:

Theorem 3.5. Let $f, p, q, g$ be as in Theorem 3.3. If $B \zeta<\theta$, then for all $\lambda \in(\zeta, \theta / B)$ the boundary value problem $\left(\left.1.2\right|_{\lambda}\right),(1.3),(1.4)$ has at least two positive solutions.

Proof. From $B \lambda<\theta$ it follows that there is a constant $m>0$ such that $f_{s}(m)<(\theta / \lambda) m$, or $F_{s}(m) \leq \theta m$. Thus from (3.3) we conclude that if $\|x\|=m$, then $\left\|A_{\lambda} x\right\| \leq\|x\|$.

Now, since $\lambda L_{0}>\varrho$, there exists $n$ such that $0<n<m$ and $\lambda f_{i}(n)>\varrho n$ or $F_{i}(n)>\varrho n$. Again, as in (3.2), we find that if $\|x\|=n$, then $\left\|A_{\lambda} x\right\|>\|x\|$.

Similarly, from the fact that $\lambda L_{\infty}>\varrho$, there is a constant $r>m$ such that $F_{i}(r)>\varrho r$. Thus as in the previous case we get $\left\|A_{\lambda} x\right\|>\|x\|$ whenever $\|x\|=r$.

Finally, we set $\Omega_{1}:=\left\{x \in C_{0}^{1}(I):\|x\|<n\right\}$ and $\Omega_{2}:=\left\{x \in C_{0}^{1}(I)\right.$ : $\|x\|<m\}$. Notice that $u<m$. Taking into account the fact that $A_{\lambda}$ is a completely continuous operator and Lemma 3.2, from Theorem 1.1, it follows that there exists a solution $x_{1}$ of the boundary value problem $\left(\left.1.2\right|_{\lambda}\right),(1.3)$, (1.4) such that $n<\left\|x_{1}\right\|<m$. Similarly, since $m<r$, there exists a solution $x_{2}$ such that $m<\left\|x_{2}\right\|<r$.

Corollary 3.6. Let $f, p, q, g$ be as in Theorem 3.3. If $\theta>B, L_{0}>\varrho$ and $L_{\infty}>\varrho$, then the boundary value problem $\left(\left.1.2\right|_{1}\right),(1.3),(1.4)$ has at least two positive solutions.

Proof. This follows from the previous theorem and the fact that $1 \in$ $(B / \theta, 1 / \zeta)$.

\section{References}

[1] R. P. Agarwal and D. O'Regan, Twin solutions to singular Dirichlet problems, J. Math. Anal. Appl. 240 (1999), 433-445.

[2] - - - Twin solutions to singular boundary value problems, Proc. Amer. Math. Soc. 128 (2000), 2085-2094.

[3] V. Anuradha, D. D. Hai and R. Shivaji, Existence results for superlinear semipositone BVP's, ibid. 124 (1996), 757-763.

[4] R. Avery, Existence of multiple positive solutions to a conjugate boundary value problem, Math. Sci. Res. Hot-Line 2 (1998), 1-6.

[5] R. Avery, J. M. Davis and J. Henderson, Three symmetric positive solutions for Lidstone problems by a generalization of the Leggett-Williams theorem, Electron. J. Differential Equations 2000, no. 40, 15 pp.

[6] R. Avery and J. Henderson, Three symmetric positive solutions for a second order boundary value problem, Appl. Math. Lett. 13 (2000), 1-7.

[7] C. J. Chyan and J. Henderson, Multiple solutions for 2mth-order Sturm-Liouville boundary value problem, Comput. Math. Appl. 40 (2000), 231-237.

[8] J. M. Davis, P. W. Eloe and J. Henderson, Triple positive solutions and dependence on higher order derivatives, J. Math. Anal. Appl. 237 (1999), 710-720. 
[9] D. R. Dunninger and H. Wang, Multiplicity of positive solutions for a nonlinear differential equation with nonlinear boundary conditions, Ann. Polon. Math. 69 (1998), $155-165$.

[10] P. W. Eloe and J. Henderson, Positive solutions and nonlinear multipoint conjugate eigenvalue problems, Electron. J. Differential Equations 1997, no. 3, 11 pp.

[11] L. H. Erbe, S. C. Hu and H. Wang, Multiple positive solutions of some boundary value problems, J. Math. Anal. Appl. 184 (1994), 640-648.

[12] L. H. Erbe and H. Wang, On the existence of positive solutions of ordinary differential equations, Proc. Amer. Math. Soc. 120 (1994), 743-748.

[13] J. Henderson and E. R. Kaufmann, Multiple positive solutions for focal boundary value problems, Comm. Appl. Anal. 1 (1997), 53-60.

[14] J. Henderson and H. B. Thompson, Multiple symmetric positive solutions for a second order boundary value problem, Proc. Amer. Math. Soc. 128 (2000), 23732379 .

[15] J. Henderson and H. Wang, Positive solutions for nonlinear eigenvalue problems, J. Math. Anal. Appl. 208 (1997), 252-259.

[16] G. L. Karakostas and P. Ch. Tsamatos, Positive solutions for a nonlocal boundary value problem with increasing response, Electron. J. Differential Equations 2000, no. 73,8 pp.

[17] - - - Multiple positive solutions for a nonlocal boundary value problem with quiet at zero response, ibid. 2001, no. 13, 11 pp.

[18] —, - On a nonlocal boundary value problem at resonance, J. Math. Anal. Appl. 259 (2001), 209-218.

[19] - - - Existence results for some n-dimensional nonlocal boundary value problems, ibid., 429-438.

[20] M. A. Krasnosel'skiǔ, Positive Solutions of Operator Equations, Noordhoff, Groningen, 1964.

Department of Mathematics

University of Ioannina

45110 Ioannina, Greece

E-mail: gkarako@cc.uoi.gr

ptsamato@cc.uoi.gr

Reçu par la Rédaction le 4.5.2001

Révisé le 28.9.2001 